\title{
BitX: Empower Versatile Inference with Hardware Runtime Pruning
}

\author{
Hongyan Li \\ Hang Lu \\ State Key Laboratory of Computer \\ Architecture, Institute of Computing \\ Technology, CAS \\ University of Chinese Academy of \\ Sciences \\ Beijing, China \\ Mingzhe Zhang \\ State Key Laboratory of Computer \\ Architecture, Institute of Computing \\ Technology, CAS \\ Beijing, China
}

\author{
Jiawen Huang
ture, Institute of C
Technology, CAS
Beijing, China \\ State Key Laboratory of Computer \\ Architecture, Institute of Computing \\ Jiawen Huang
Technology, CAS
Beijing, China \\ Jiawen Huang
Laboratory of Co, Institute of C
Technology, CAS
Beijing, China
}

Wei Chen

State Key Laboratory of Computer

Architecture, Institute of Computing

Technology, CAS

Beijing, China

Xiaowei Li

State Key Laboratory of Computer

Architecture, Institute of Computing

Technology, CAS

University of Chinese Academy of

Sciences

Beijing, China

\author{
Wenxu Wang \\ State Key Laboratory of Computer \\ Architecture, Institute of Computing \\ Technology, CAS \\ University of Chinese Academy of \\ Sciences \\ Beijing, China
}

Liang Chang

University of Electronic Science and

Technology of China

Chengdu, China

\begin{abstract}
Classic DNN pruning mostly leverages software-based methodologies to tackle the accuracy/speed tradeoff, which involves complicated procedures like critical parameter searching, fine-tuning and sparse training to find the best plan. In this paper, we explore the opportunities of hardware runtime pruning and propose a hardware runtime pruning methodology, termed as "Bit $X$ " to empower versatile DNN inference. It targets the abundant useless bits in the parameters, pinpoints and prunes these bits on-the-fly in the proposed BitX accelerator. The versatility of BitX lies in: (1) software effortless; (2) orthogonal to the software-based pruning; and (3) multi-precision support (including both floating point and fixed point). Empirical studies on image classification and object detection models highlight the following results: (1) up to 4.82x speedup over the original non-pruned DNN and 14.76x speedup collaborated with the software-pruned DNN; (2) up to $0.07 \%$ and $0.9 \%$ higher accuracy for the floating-point and fixed-point DNN, respectively; (3) $2.00 \mathrm{x}$ and $3.79 \mathrm{x}$ performance improvement over the state-of-theart accelerators, with $0.039 \mathrm{~mm}^{2}$ and $68.62 \mathrm{~mW}$ (floating-point 32),

Permission to make digital or hard copies of all or part of this work for personal or classroom use is granted without fee provided that copies are not made or distributed for profit or commercial advantage and that copies bear this notice and the full citation on the first page. Copyrights for components of this work owned by others than ACM must be honored. Abstracting with credit is permitted. To copy otherwise, or republish, to post on servers or to redistribute to lists, requires prior specific permission and/or a fee. Request permissions from permissions@acm.org.

ICPP '21, August 9-12, 2021, Lemont, IL, USA

(C) 2021 Association for Computing Machinery.

ACM ISBN 978-1-4503-9068-2/21/08 _\$15.00

https://doi.org/10.1145/3472456.3472513
\end{abstract}

$36.41 \mathrm{~mW}$ (16-bit fixed point) power consumption under TSMC 28 nm technology library.

\section{CCS CONCEPTS}

- Computer systems organization $\rightarrow$ Neural networks.

\section{KEYWORDS}

hardware pruning, deep learning accelerator, neural networks

ACM Reference Format:

Hongyan Li, Hang Lu, Jiawen Huang, Wenxu Wang, Mingzhe Zhang, Wei Chen, Liang Chang, and Xiaowei Li. 2021. BitX: Empower Versatile Inference with Hardware Runtime Pruning. In 50th International Conference on Parallel Processing (ICPP '21), August 9-12, 2021, Lemont, IL, USA. ACM, New York, NY, USA, 12 pages. https://doi.org/10.1145/3472456.3472513

\section{INTRODUCTION}

Large computation intensity is well recognized as one of the main obstacles to deploy DNNs into practical applications, because of the rapid evolution of the parameter size from millions (i.e. ResNet [10] family in computer vision) to even hundreds of billions (i.e. BERT [7] or GPT-3 [5] in natural language processing). Although more complex models with enormous layers and complicated neuron connections will benefit the ever-increasing accuracy demand, the real-time performance enhancement, which is the more important

Author 1 and 2 contributed equally. Corresponding author is Hang Lu, email: luhang@ict.ac.cn 
Table 1: WEIGHT/BIT sparsity comparison for various DNNs pre-trained with ImageNet dataset. Bit sparsity is significantly more abundant than weight sparsity. The weights are represented by floating-point 32 .

\begin{tabular}{lll}
\hline Model & Weight Sparity & Bit Sparity \\
\hline DenseNet121 & $4.84 \%$ & $48.64 \%$ \\
ResNet50 & $0.33 \%$ & $48.64 \%$ \\
ResNet152 & $0.75 \%$ & $48.64 \%$ \\
ResNext50_32x4d & $0.37 \%$ & $48.64 \%$ \\
ResNext101_32x8d & $3.43 \%$ & $48.65 \%$ \\
InceptionV3 & $0.05 \%$ & $48.64 \%$ \\
MNASNet0.5 & $0.00 \%$ & $48.60 \%$ \\
MNASNet1.0 & $8.07 \%$ & $48.98 \%$ \\
MobileNetV2 & $0.01 \%$ & $48.67 \%$ \\
ShuffleNetV2_x0_5 & $0.00 \%$ & $48.36 \%$ \\
ShuffleNetV2_x1_0 & $1.53 \%$ & $48.63 \%$ \\
SqueezeNet1_0 & $0.05 \%$ & $48.64 \%$ \\
SqueezeNet1_1 & $0.02 \%$ & $48.64 \%$ \\
\hline
\end{tabular}

and desirable request however, cannot catch up with the development of DNNs, especially for the handhelds and cyber physical devices.

Pruning is universally accepted as an effective way in maintaining the model accuracy and optimizing the computation intensity at the same time. Almost all the conventional pruning methodologies, i.e. [11] [21] [25] [22] [33] rely on software-level efforts which usually consist of the following steps: evaluate the importance of neurons, remove the least important fraction of neurons (contingent to the preset compression ratio), parameter fine-tuning until satisfaction, or get unsatisfactory accuracy that has to change the importance metric and commence pruning again. Generally speaking, software-based pruning has competitive advantages in (1) obtaining maintained accuracy and controllable compression ratio, and (2) easy deployment without considering the underlying hardware (for structured pruning of course). Due to the diversity of deep learning applications, however, it is almost impossible to find a universal software-based pruning method that is applicable to all use cases. A direct consequence is that end-users must reconsider the application-specific pruning criteria, in terms of the superparameters and DNN structured parameters and re-implement the above steps from the very inception. The tediously repeated effort limits the fast deployment of DNNs in the practical use.

From the model perspective, the DNN itself, or its internal sparsity level also impairs the software-based pruning. In specific, pruning leverages the importance metric to identify the least contributive parameters. The metric measures the sparsity variants of the weights or activations, i.e. the average percentage of zeros [11], the absolute value of filters [21], or the entropy of filters [25] and so on, trying to eliminate the zero or near-zero variants and retrain the model until the optimal accuracy to justify the employed importance metric. However, one metric may suit for certain DNNs very well but might not behave perfectly for others. Besides, the headroom of the sparsity is not always adequate either. Some pruning approaches have to commence retraining to compensate the information loss or sparse training to manually create more sparsity [22] [33] [4] in
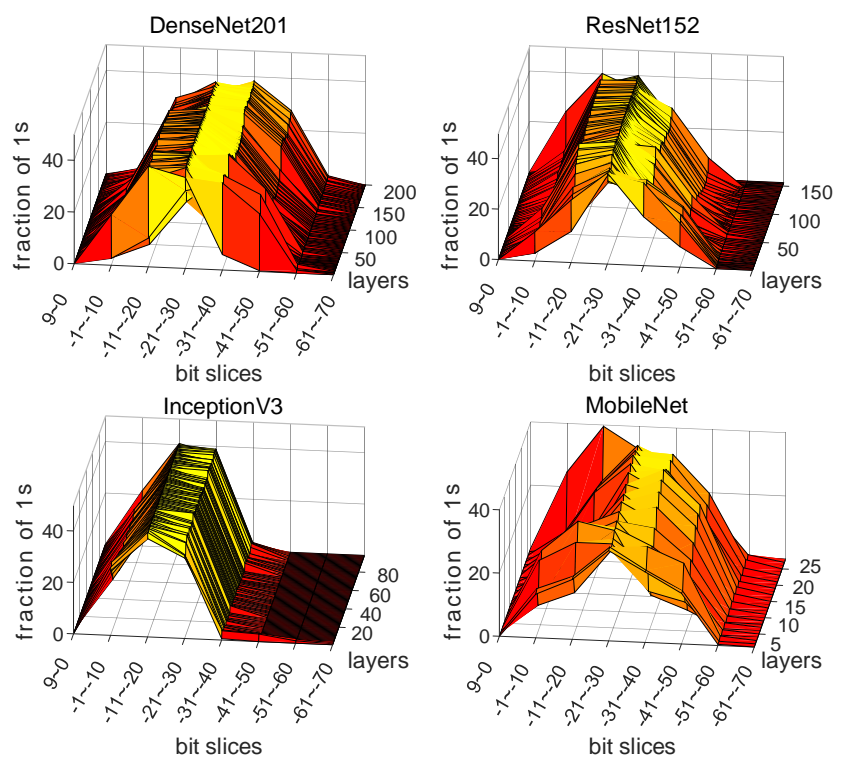

Figure 1: Distribution analysis of bit 1s. The 4 benchmark DNNs demonstrate a similar behavior: the surfplot reaches its peak at $2^{-21} \sim 2^{-30}$, which means this bit slice has the largest fraction of bit 1s (nearly $40 \%$ ), but most of them are trivial. BitX aims to prune these trivial bits to obtain the inference acceleration.

the parameter set, which is even more time-consuming and laborintensive.

From the efficiency perspective, the labor intensity of the softwarebased pruning also exhibits in the parameter fine-tuning phase. That is because the remaining non-pruned weights cannot always guarantee the initial accuracy of the DNN. Classic procedure hence relies on retraining to redeem the lost accuracy with the same dataset and time-consuming iterations that usually cost days or even weeks according to the equipped GPU facility. The above procedure is usually implemented layer-wise, so if we apply it to VGG-19 [29] for example, we need to retrain the model 19 times with each time iterating tens of epochs to recover the lost accuracy. The long and tedious retraining prevents the instant deployment of the pruned model into the devices, and worse still, if the accuracy is not satiable, it must repeat the same tedious procedure again. Considering other widely used DNNs with hundreds of layers (i.e. ResNet [10], DenseNet [12]) or even much larger and more complex connections like 3D convolution [31], non-local convolution [32] or deformable convolution [6], the developers therefore usually face a formidable challenge to obtain both the satisfactory result and the shorter time spent.

From the accelerator perspective, unstructured pruning relies heavily on the underlying hardware. There are plenty of accelerator prototypes proposed to support the particular pruning methodology. For example, Cambricon-S [34] addresses the irregularity of unstructured pruning. EIE [30] supports the pruning only for the fully-connected layers; ESE [9] only focuses on the sparse LSTM model, while the convolutional layers that dominate the $\mathrm{CNN}$ inference computation are not supported. Accelerator design also depends on various sparsification methodologies. SCNN [27] exploits 


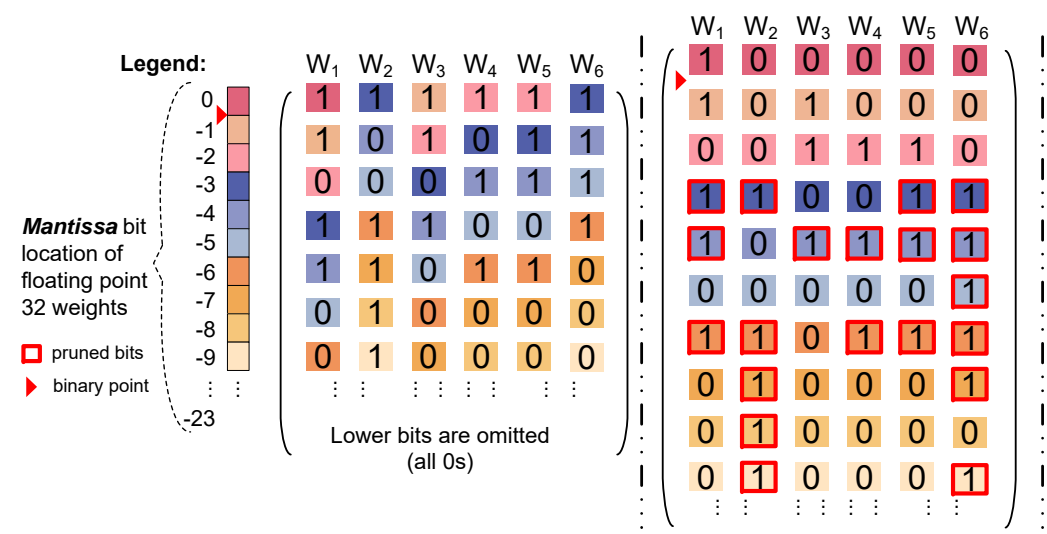

(a) original weight matrix

(b) exponents alignment

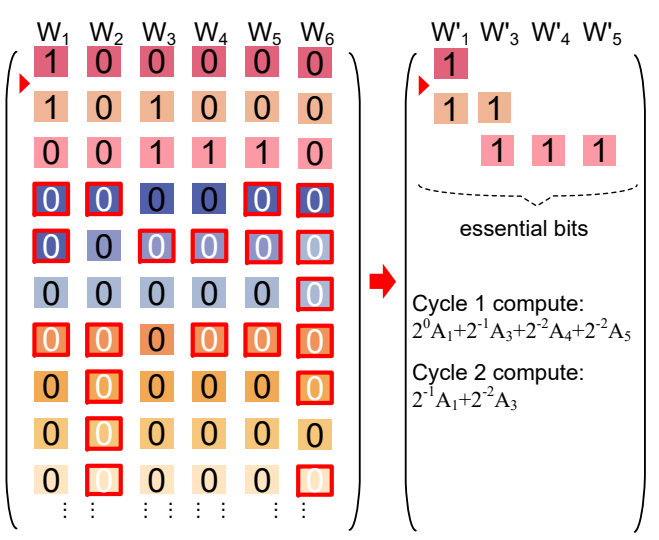

(c) bit slice extraction

Figure 2: Core concept of BitX. Bit matrix before pruning is shown in (a). (b) demonstrates exponent alignment according to IEEE 754. Six non-essential bit rows are pruned as shown in (c), only leaving a more compact essential-bit matrix.

the neuron and synapse sparsity, while Cnvlutin [3] only supports neuron sparsity. If the software engineer modifies the pruning policy or simply changes from structured pruning to the unstructured pruning, the hardware employed is also about to change, attached with overwhelming transplantation overhead.

Ideally, a pre-trained DNN should be pruned as fast as possible for the timely deployment in hardware, and more desirably, the hardware could directly implement runtime pruning without any tedious software-level work to accelerate the DNN inference in a handy and efficient manner. This necessity stimulates us to reconsider the existing classic pruning methodologies and explore a new style to free the developers from the labor-intensive software effort. Therefore, in this paper, we propose BitX, a hardware runtime pruning methodology to empower versatile DNN inference. Apart from the software-based pruning that requires complex algorithm to identify the trivial values by repeated trial-and-error, BitX implements pruning by targeting bits. It aims to pinpoint the essential bits and prune away the useless bits in the parameters, because the useless bits are more easily exposed especially at the hardware level. For example, the floating-point value has long bit-width in its mantissa (24 bits) [13], and the exponent always shifts the mantissa to align the binary point with another value for computation. The shifted binary positions are automatically zero-padded and involved in the floating-point arithmetic. This procedure generates two types of useless bits: the $1^{\text {st }}$ type is the genetic zero bits in the mantissa and the automatically padded zero bits; the $2^{\text {nd }}$ type is more implicit, that is, the rear bit $1 \mathrm{~s}$ with extremely trivial significance. As will be shown in Section 2, the two types of useless bits both occupy a large fraction in the binary represented weights, which also provides a decent condition for BitX to prune these bits directly in the accelerator at the inference runtime.

Our evaluations have shown that by precisely locating and pruning these useless bits, the inference speed could be significantly boosted (Section 4.2). Most importantly, the whole pruning operation could be implemented on-the-fly in the proposed BitX accelerator, with lossless accuracy and without any software related effort.

The contributions of this paper are listed as follows:
- We propose a novel hardware runtime pruning method, termed as BitX, to empower versatile DNN inference. We highlight the following features of BitX:

(1) Software effortless: BitX directly prunes the original DNN. No retraining, fine-tuning, or special library/framework needed, because it targets the useless binary bits not values.

(2) Orthogonal to the existing software pruning methodologies: BitX implements straightforward bit pruning in the accelerator, so the DNNs, either pruned or nonpruned at the software level, are all suitable for BitX. In other words, it could further prune the useless bits of the software-pruned DNN, and obtain additional speedup.

(3) Multi-precision support: BitX applies to not only floatingpoint but also fixed-point DNNs. The fixed-point DNNs also demonstrate substantial useless bits. BitX could accelerate these models with even higher accuracy and speedup.

- We propose a deep learning accelerator capable of unprecedented hardware runtime pruning to mine the maximum potential of BitX. We highlight the following results:

(1) Speedup: two representatives of BitX accelerator - BitXmild and BitX-wild could respectively obtain $2.61 \mathrm{x} \sim 4.82 \mathrm{x}$ faster speed over the non-pruned baselines under floatpoint 32 mode, and up to $2.00 \mathrm{x}$ under 16-bit fixed-point mode. For the object detection model YoloV3, the speedup is up to $4.98 \mathrm{x}$ and $14.76 \mathrm{x}$ higher over the original model.

(2) Accuracy: for ImageNet, the accuracy loss is $0.13 \%$ and $0.44 \%$ for BitX-mild and BitX-wild; for Cifar-10, the loss is $0.09 \%$ and $0.15 \%$. The above accuracy data are reported by the floating-point DNNs. Under 16-bit fixed-point, the accuracy is even $0.9 \%$ and $0.2 \%$ higher than the baseline DenseNet121 and ResNext101 for BitX-mild; 0.8\% and 0.1\% higher for BitX-wild. For YoloV3, the accuracy is $0.06 \%$ and $0.07 \%$ higher than the original model for BitX-mild; $0.31 \%$ and $1.64 \%$ lower for BitX-wild.

(3) Accelerator Performance: we compare the BitX accelerator performance with other state-of-the-art accelerator 
prototypes. Equipped by BitX, the accelerator achieves $2.00 \mathrm{x}$ and $3.79 \mathrm{x}$ performance improvement. The area is $0.039 \mathrm{~mm}^{2}$ and $68.62 \mathrm{~mW}$ (floating-point 32 ) and $36.41 \mathrm{~mW}$ (16-bit fixed point) under TSMC $28 \mathrm{~nm}$ technology library.

(4) Sensitivity: we thoroughly evaluate the sensitivity of the key design parameters to the accuracy and speed (see Section 4.3).

Powered by the above features, BitX is designed for flexible and versatile DNN inference at any circumstances. In the next section, we will start from discussing two key observations that justify BitX.

\section{OPPORTUNITIES OF HARDWARE RUNTIME PRUNING}

\subsection{Bit-level Sparsity $-1^{\text {st }}$ target}

For most of the software-based pruning approaches in the literature [22] [11] [26] [33], the classic procedure basically involves identifying and pruning the trivial "near-zero" parameters. However as mentioned above, the headroom of the value-level sparsity is very limited. If the compression ratio is mistakenly set, the accuracy loss is inevitable. Under such circumstances, two alternatives are always considered: lower the compression ratio and roll back to the inception [11] [26], or commence sparse training to create more headroom for the employed pruning metric [22] [33]. It is also the root reason that the labor-intensive software effort stems from.

In order to circumvent the inconvenience, we re-examine the parameters in-depth. Instead of sticking to the sparse "values", we analyze the more fine-grained bit-level sparsity. As shown in Table 1 , the "weight sparsity" proportion is obtained by counting the values below $10^{-5}$ over the total parameter size, while the "bit sparsity" proportion is by counting total bit 0 s over the total "bit count" of the mantissas in the parameter set. Obviously, various benchmark DNNs uniformly demonstrate an obvious gap between the two sides. Most of the weight sparsity results are less than $1 \%$. The bit sparsity however, are nearly $49 \%$ and no exception. It provides an excellent opportunity of exploiting the sparsity at the bit level without resorting to the value pruning, because $\sim 49 \%$ bits are already 0 s and removing these useless bits off the MAC computation is definitely harmless to the accuracy. BitX intends to fully utilize this decent condition to accelerate the DNN inference.

\subsection{Trivial bit $1 \mathrm{~s}-2^{\text {nd }}$ target}

Obviously, we can design particular zero-skipping mechanism to avoid the ineffectual computations caused by the zero bits, which is also the main objective many previous sparsity-aware acceleration schemes target [23] [24] [2] [19]. However, the trivial "bit 1s", as another factor that influences the inference efficiency, are barely considered but they are exactly the major optimization objective in BitX. Therefore, having explored the bit-level "sparsity" (or the fraction of bit 0s), we further migrate our focus to the useless "bit $1 \mathrm{~s}$ ". The $49 \%$ fraction of $0 \mathrm{~s}$ also means the percentage of bit $1 \mathrm{~s}$ is around $51 \%$, which is also a very large fraction. More importantly, not all the bit $1 \mathrm{~s}$ are influential to the final accuracy. If we could identify the "essential" bit 1 s and prune away the trivial ones, the inference efficiency could be further boosted.

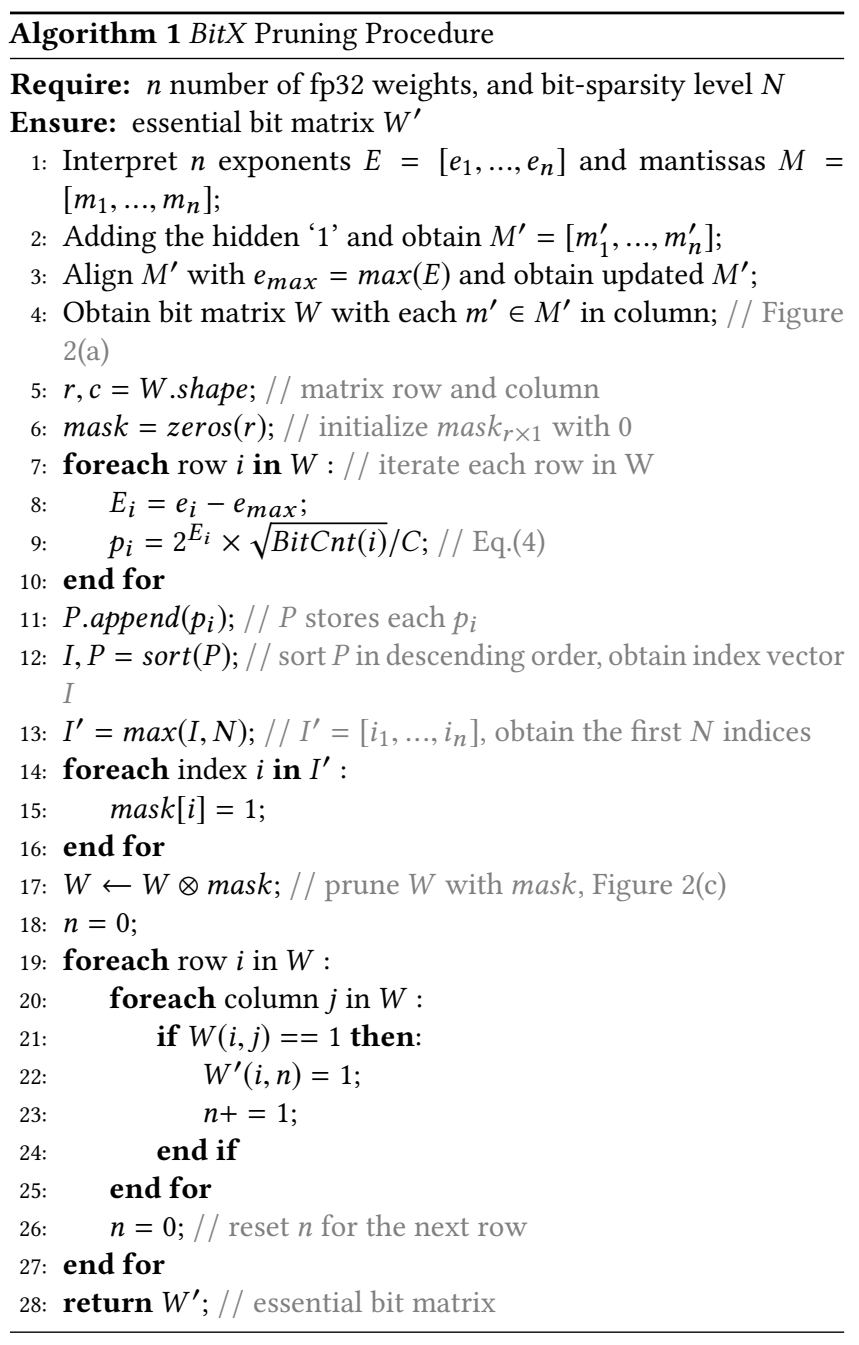

As evidence, we explore the distribution of bit $1 \mathrm{~s}$ in each bit slice. As shown in Figure 1, the X-axis denotes the bit slice of the binary represented weight (in floating-point 32). Each bit slice reflects the significance of the bits. For example, if a dummy weight is $1.1101 \times 2^{-4}$, its binary representation is 0.00011101 and we record the significance of the four bit $1 \mathrm{~s}$ are the $2^{-4}, 2^{-5}, 2^{-6}$, and $2^{-8}$ after the binary point, respectively.

According to Figure 1, the bit slice could range from bit significance " $9 \sim 0$ " before the binary point to "-61 -70" after the binary point. All the evaluated DNNs exhibit an "arched" shape across each layer on the Y-axis. The central bit slices own most of the bit 1s ( 40\%), i.e. ResNet152 and DenseNet201. Taking bit significance $2^{-21} \sim 2^{-30}$ as the representative, the equivalent decimals are in range: $0.000000477\left(\sim 10^{-8}\right)$ to $0.000000000931\left(\sim 10^{-11}\right)$. These tiny values are very likely to be less contributive to the final accuracy. Therefore, BitX aims to precisely identify the essential bits and prune the large fraction of the trivial bits on the fly in the accelerator, to reduce the computation intensity under the constraint of tiny accuracy loss. In the next section, we will elaborate how it is designed to achieve this objective. 

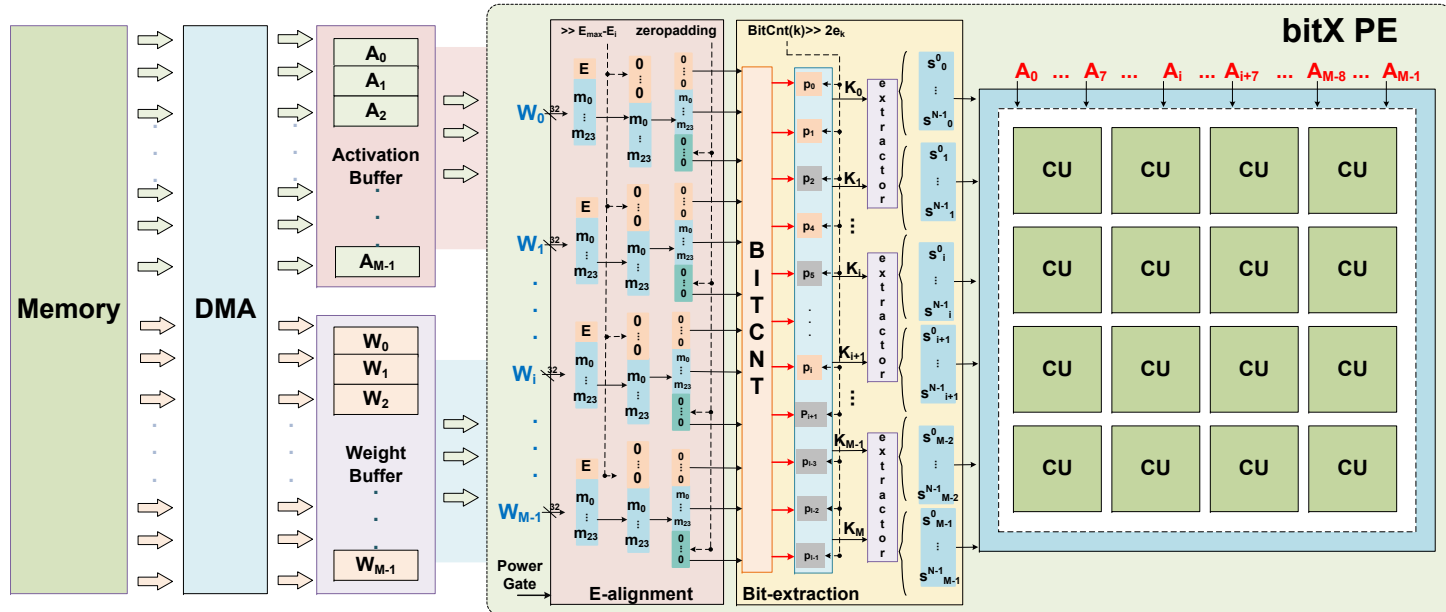

Figure 3: Overview architecture of the BitX accelerator.

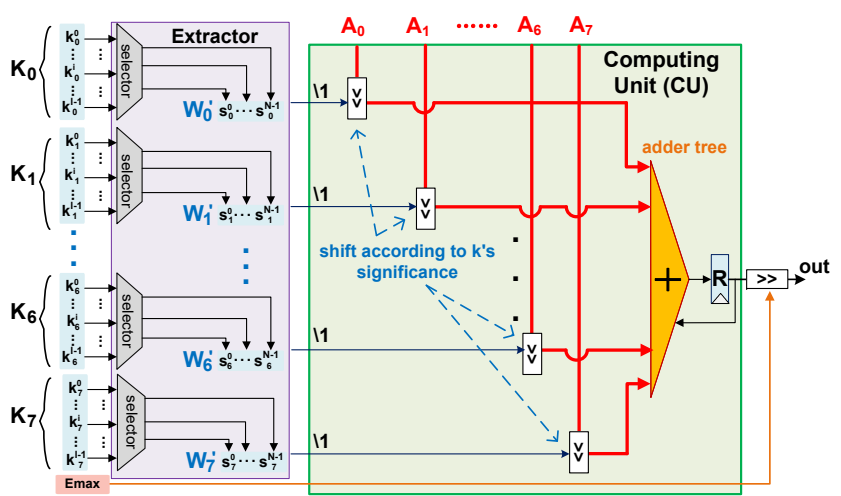

Figure 4: Microarchitecture of the "compute unit" (CU).

\section{BITX}

\subsection{General Concept}

Without losing generality, a floating-point operand is composed of three portions: the signed bit, mantissa, and exponent, following IEEE 754 [13] which is also the most commonly used floating-point standard in industry. If we employ the single precision (fp32) format, the mantissa comprises 23 bits and the exponent occupies 8 bits with the last bit for the sign. A single precision weight fp could be expressed as $f_{p}=(-1)^{s} 1 . m \times 2^{e-127}$, in which $e$ is the actual position of the "binary point" plus 127 .

If we take 6 non-aligned fp32 weights as an example and interpret their mantissas as illustrated in Figure 2(a), we get a bit matrix with each column showing the binary mantissa actually stored in memory. Different colors in the legend indicate the bit significance from $2^{-1}$ to $2^{-9}$ after the binary point (position 0 denotes the hidden 1 of the mantissa [13]). In terms of the exponent, we use different background colors in the bit matrix to indicate the actual significance of this bit guided by the exponent. For example, the topmost bit 1 marked as dark blue in $W_{2}$ is actually the $2^{-3}$ significance in the fractional part.
If we align the mantissa according to the exponent, zeros are padded in the front vacancies just as shown in Figure 2(b). The first observation is that the aforementioned bit-level sparsity is more abundant after zero padding, which provides excellent condition for the bit-level pruning. The second observation is that a large fraction of bit $1 \mathrm{~s}$ are shifted to the rear direction beyond $2^{-6}$ significance. A direct consequence is that the practical contribution of these bits are pretty trivial to the final MAC. If we could prune away these insignificant $1 \mathrm{~s}$, it could save plenty of bit-level arithmetic and speed up the inference. As illustrated in Figure 2(c), the red box denotes the pruned bit $1 \mathrm{~s}$, only leaving several essential $1 \mathrm{~s}$ to form the pruned weights: $W_{1}{ }^{\prime}, W_{3}{ }^{\prime}, W_{4}{ }^{\prime}$ and $W_{5}{ }^{\prime}$, and we term these $1 \mathrm{~s}$ as the "essential bits".

\subsection{Methodology}

Leveraging the essential bits in Figure 2(c) is an effective way to simplify a series of value-level MACs as bit-level additions [23] [20]. However, for the millions of parameters in DNN, if we use fp32 to represent these values, the impact of a single bit to the whole network is not that easy to be determined. The leftover problem is how to create an effective yet hardware-friendly mechanism to make full use of the abundant useless bits and maintain the initial accuracy, without labor-intensive software tricks. In this subsection, we firstly formalize the problem and then elaborate the BitX procedure.

3.2.1 Problem Formulation. Given an $n \times l$ matrix $A$ (activations) and an $l \times n$ matrix $W$ (weights), the result of $A \times W$ can be represented by the summation of $\mathrm{n}$ rank-one matrices: $A^{(i)}$ represents the i-th row of $A$ and $W_{(i)}$ for the $i$-th column of $W$, as shown in Eq. (1). The criticality of these rank-one matrices could be easily decided by the Fast Monte-Carlo Algorithm [8], in which some rank-one matrices are randomly sampled to approximate $A \times W$. The most common sampling method [8] to select these rank-one matrices is by referring to their respective probability as shown in Eq. (2). It is obtained by computing the Euclidean distance of 
Table 2: Cifar-10 performance. The last 'Avg.' row denotes the "accuracy loss / sparsity increment". The accuracy loss is obtained by itemizing the accuracy loss of each benchmark model versus the original and calculate their average(in \%). The sparsity increment is obtained by counting the bit 0 s after pruning and normalizing the data to the original (in $x$ ).

\begin{tabular}{llllll}
\hline Model & Original & $\mathbf{N}=\mathbf{1 0}$ & $\mathbf{N}=\mathbf{8}$ & $\mathbf{N}=\mathbf{6}$ & $\mathbf{N}=\mathbf{4}$ \\
\hline DenseNet121 & $95.25 / 1 \mathrm{x}$ & $95.21 / 1.36 \mathrm{x}$ & $95.19 / 1.49 \mathrm{x}$ & $95.10 / 1.63 \mathrm{x}$ & $93.17 / 1.77 \mathrm{x}$ \\
DenseNet161 & $95.66 / 1 \mathrm{x}$ & $95.55 / 1.33 \mathrm{x}$ & $95.52 / 1.47 \mathrm{x}$ & $95.52 / 1.61 \mathrm{x}$ & $93.98 / 1.76 \mathrm{x}$ \\
DenseNet169 & $95.50 / 1 \mathrm{x}$ & $95.48 / 1.33 \mathrm{x}$ & $95.49 / 1.47 \mathrm{x}$ & $95.44 / 1.61 \mathrm{x}$ & $93.28 / 1.76 \mathrm{x}$ \\
Densenet201 & $95.35 / 1 \mathrm{x}$ & $95.39 / 1.31 \mathrm{x}$ & $\mathbf{9 5 . 3 5 / 1 . 4 5 x}$ & $95.25 / 1.60 \mathrm{x}$ & $93.71 / 1.75 \mathrm{x}$ \\
ResNet18 & $95.18 / 1 \mathrm{x}$ & $94.96 / 1.66 \mathrm{x}$ & $94.90 / 1.75 \mathrm{x}$ & $94.80 / 1.83 \mathrm{x}$ & $93.69 / 1.91 \mathrm{x}$ \\
ResNet34 & $95.33 / 1 \mathrm{x}$ & $95.27 / 1.66 \mathrm{x}$ & $95.27 / 1.74 \mathrm{x}$ & $95.20 / 1.83 \mathrm{x}$ & $93.69 / 1.91 \mathrm{x}$ \\
ResNet50 & $95.14 / 1 \mathrm{x}$ & $95.07 / 1.42 \mathrm{x}$ & $95.04 / 1.53 \mathrm{x}$ & $95.07 / 1.66 \mathrm{x}$ & $91.79 / 1.78 \mathrm{x}$ \\
ResNet101 & $95.51 / 1 \mathrm{x}$ & $95.34 / 1.44 \mathrm{x}$ & $95.35 / 1.56 \mathrm{x}$ & $95.39 / 1.69 \mathrm{x}$ & $91.46 / 1.81 \mathrm{x}$ \\
ResNet152 & $95.56 / 1 \mathrm{x}$ & $95.41 / 1.45 \mathrm{x}$ & $95.47 / 1.57 \mathrm{x}$ & $95.36 / 1.69 \mathrm{x}$ & $90.48 / 1.81 \mathrm{x}$ \\
ResNext29_2x64d & $95.82 / 1 \mathrm{x}$ & $95.71 / 1.45 \mathrm{x}$ & $95.73 / 1.57 \mathrm{x}$ & $95.71 / 1.69 \mathrm{x}$ & $91.95 / 1.81 \mathrm{x}$ \\
ResNext29_4x64d & $95.69 / 1 \mathrm{x}$ & $95.57 / 1.37 \mathrm{x}$ & $95.55 / 1.50 \mathrm{x}$ & $95.50 / 1.64 \mathrm{x}$ & $92.82 / 1.78 \mathrm{x}$ \\
ResNext29_8x64d & $96.19 / 1 \mathrm{x}$ & $96.13 / 1.31 \mathrm{x}$ & $96.16 / 1.45 \mathrm{x}$ & $96.08 / 1.60 \mathrm{x}$ & $93.57 / 1.75 \mathrm{x}$ \\
ResNext29_32x64d & $95.61 / 1 \mathrm{x}$ & $95.56 / 1.25 \mathrm{x}$ & $95.55 / 1.40 \mathrm{x}$ & $95.48 / 1.56 \mathrm{x}$ & $89.47 / 1.73 \mathrm{x}$ \\
Avg. loss/sparsity & $\mathbf{0 . 0 0 0 / 1 x}$ & $\mathbf{0 . 0 9 0 / 1 . 4 1 x}$ & $\mathbf{0 . 0 9 4 / 1 . 5 3 x}$ & $\mathbf{0 . 1 4 5 / 1 . 6 6 x}$ & $\mathbf{2 . 9 7 9 / 1 . 8 0 x}$ \\
\hline
\end{tabular}

Table 3: ImageNet performance. The computing method is identical to Table 2.

\begin{tabular}{llllll}
\hline Model & Original & $\mathbf{N = 1 0}$ & $\mathbf{N = 8}$ & $\mathbf{N = 6}$ & $\mathbf{N = 4}$ \\
\hline DenseNet121 & $71.96 / 1 \mathrm{x}$ & $71.95 / 1.34 \mathrm{x}$ & $71.00 / 1.47 \mathrm{x}$ & $71.00 / 1.62 \mathrm{x}$ & $65.00 / 1.76 \mathrm{x}$ \\
DenseNet161 & $75.28 / 1 \mathrm{x}$ & $75.20 / 1.32 \mathrm{x}$ & $75.14 / 1.46 \mathrm{x}$ & $74.79 / 1.61 \mathrm{x}$ & $72.00 / 1.76 \mathrm{x}$ \\
DenseNet169 & $73.75 / 1 \mathrm{x}$ & $73.56 / 1.31 \mathrm{x}$ & $73.55 / 1.45 \mathrm{x}$ & $73.55 / 1.60 \mathrm{x}$ & $68.62 / 1.75 \mathrm{x}$ \\
Densenet201 & $74.56 / 1 \mathrm{x}$ & $74.46 / 1.30 \mathrm{x}$ & $74.40 / 1.44 \mathrm{x}$ & $74.24 / 1.59 \mathrm{x}$ & $69.00 / 1.74 \mathrm{x}$ \\
ResNet18 & $67.28 / 1 \mathrm{x}$ & $67.09 / 1.64 \mathrm{x}$ & $67.00 / 1.73 \mathrm{x}$ & $66.72 / 1.81 \mathrm{x}$ & $62.52 / 1.90 \mathrm{x}$ \\
ResNet34 & $71.32 / 1 \mathrm{x}$ & $71.11 / 1.65 \mathrm{x}$ & $71.10 / 1.73 \mathrm{x}$ & $70.92 / 1.82 \mathrm{x}$ & $68.00 / 1.90 \mathrm{x}$ \\
ResNet50 & $74.50 / 1 \mathrm{x}$ & $74.50 / 1.41 \mathrm{x}$ & $74.51 / 1.54 \mathrm{x}$ & $74.10 / 1.67 \mathrm{x}$ & $67.00 / 1.80 \mathrm{x}$ \\
ResNet101 & $76.00 / 1 \mathrm{x}$ & $76.06 / 1.43 \mathrm{x}$ & $76.05 / 1.55 \mathrm{x}$ & $75.76 / 1.68 \mathrm{x}$ & $69.02 / 1.81 \mathrm{x}$ \\
ResNet152 & $77.02 / 1 \mathrm{x}$ & $76.56 / 1.44 \mathrm{x}$ & $76.55 / 1.56 \mathrm{x}$ & $76.46 / 1.69 \mathrm{x}$ & $72.30 / 1.81 \mathrm{x}$ \\
ResNext50_32x4d & $76.29 / 1 \mathrm{x}$ & $75.99 / 1.24 \mathrm{x}$ & $75.96 / 1.39 \mathrm{x}$ & $75.67 / 1.56 \mathrm{x}$ & $65.01 / 1.72 \mathrm{x}$ \\
ResNext101_32x8d & $78.24 / 1 \mathrm{x}$ & $78.20 / 1.27 \mathrm{x}$ & $78.30 / 1.42 \mathrm{x}$ & $78.10 / 1.58 \mathrm{x}$ & $73.00 / 1.74 \mathrm{x}$ \\
SqueezeNet1_1 & $54.84 / 1 \mathrm{x}$ & $\mathbf{5 4 . 8 6 / 1 . 4 2 x}$ & $54.70 / 1.54 \mathrm{x}$ & $54.40 / 1.67 \mathrm{x}$ & $47.30 / 1.80 \mathrm{x}$ \\
Avg. loss / sparsity & $\mathbf{0 . 0 0 0 / 1 x}$ & $\mathbf{0 . 1 3 1 / 1 . 4 0 x}$ & $\mathbf{0 . 2 4 2 / 1 . 5 2 x}$ & $\mathbf{0 . 4 4 4 / 1 . 6 6 x}$ & $\mathbf{6 . 0 2 3 / 1 . 7 9 x}$ \\
\hline
\end{tabular}

$A^{(i)}$ and $W_{(i)}$, which reflects the importance of the rank-one matrix multiplication.

Inspired by the Fast Monte-Carlo Algorithm, we enroll the same probability concept in BitX to measure the importance of the weight bits instead of values. Bits with smaller probability tends to play a trivial role when multiplied with the activations compared with other more important bits in the same weight. Therefore, we abstract the bit matrix in Figure 2(a) as $W$ and our objective is seeking out the (in)significant bit rows in Figure 2(b) and simplifying MAC computations. The problem remains how we can utilize the probability in Eq. (1) to sample each bit row in $W$, and determine the to-be pruned bit rows.

$$
p_{i}=\frac{\left|A^{(i)}\right|\left|W_{(i)}\right|}{\sum_{i^{\prime}=1}^{l}\left|A^{\left(i^{\prime}\right)}\right|\left|W_{\left(i^{\prime}\right)}\right|}
$$

3.2.2 Bit-slice Extraction. In $W$ (Figure 2 (a)), we target the mantissa of $n$ normal floating-point 32 weights. Each mantissa is instantiated as a column vector comprised of its bits. Obviously, $n$ weights are associated with the same number of activations for MAC. $N$ activations consist of another column vector $\left[A_{1}, A_{2} \ldots A_{j} \ldots A_{n}\right]^{T}$. We put the two column vectors into Eq. (1), so it could be rewritten as Eq. (2):

$$
p_{i}=\frac{\left|A^{(i)}\right| \times \sqrt{\sum_{j=1}^{n}\left(2^{\left.E_{i}^{j} \times v_{j}\right)^{2}}\right.}}{\sum_{i^{\prime}=1}^{l}\left(\left|A^{\left(i^{\prime}\right)}\right| \times \sqrt{\sum_{j=1}^{n}\left(2^{E_{i^{\prime}}^{j}} \times v_{j}\right)^{2}}\right)}
$$

$A_{j}$ is the element of the activation vector, and $v_{j}$ is the $j$-th bit of the $i$-th row vector in the bit matrix $W$. Each bit in the same row $i$ has its own exponent, so we use $2^{E_{i}^{j}}$ in Eq. (2) to represent the 


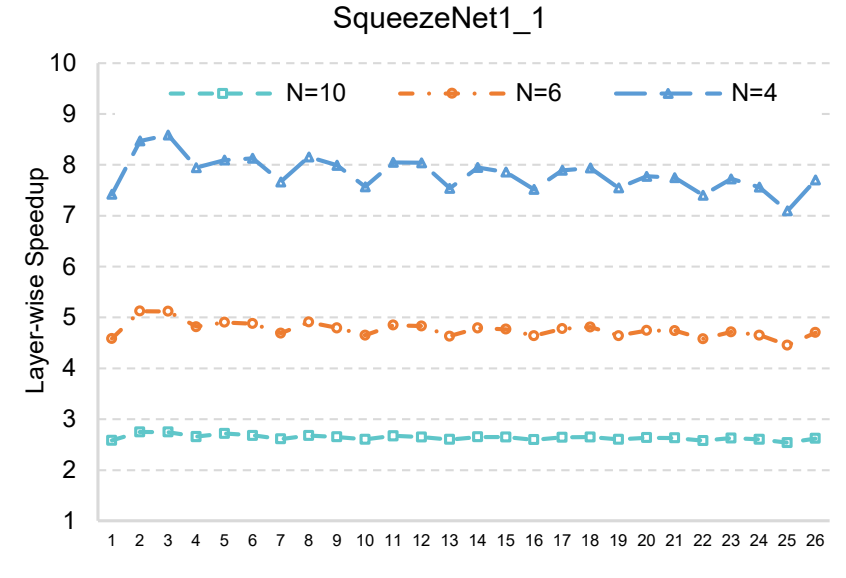

layers

Figure 5: layer-wise speedup for ImageNet dataset. The speedup of the original model is regarded as 1 on the Y-axis.

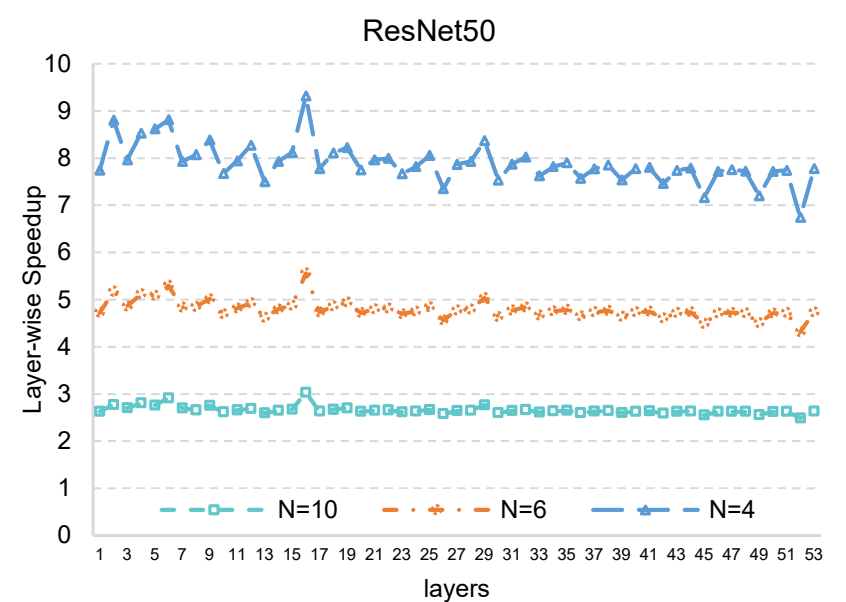

Figure 6: the method is the same as Figure 5. Higher is better.

exponent at position $j$. The Euclidean distance of the row vector is calculated as $\sqrt{\sum_{j=1}^{n}\left(2^{E_{i}^{j}} \times v_{j}\right)^{2}}$.

In BitX, exponent alignment procedure is almost identical to the normal floating-point addition [13], except one special difference, that is, BitX does not implement weight/activation MAC one by one. Instead, it aligns a group of weights simultaneously to the maximum exponent. Therefore, after exponent matching the bits in the same row $i$ share the same exponent just as Figure 2(b) has shown, and we use a uniform $E_{i}$ to denote the actual exponent of the row $i$.

$v$ is a row vector in $W$ composed of bits. For the case that a bit element $v_{j}$ equals to 0 , there is obviously no impact on calculating the Euclidean distance and thus no impact on $p_{i}$. Therefore, acquiring the Euclidean distance is equivalent to counting the number of bit $1 \mathrm{~s}$ in row $i$. We use BitCnt $(i)$ to indicate such operation, so the probability $p_{i}$ of the $i$-th row can be represented as Eq. (3):

$$
\begin{aligned}
p_{i} & =\frac{\left|A^{(i)}\right| \times \sqrt{\left(2^{E_{i}}\right)^{2} \times \operatorname{BitCnt}(i)}}{\left|A^{\left(i^{\prime}\right)}\right| \times \sum_{i^{\prime}=1}^{l}\left(\sqrt{\left(2^{E_{i^{\prime}}}\right)^{2} \times \operatorname{BitCnt}\left(i^{\prime}\right)}\right)} \\
& =\frac{\sqrt{\left(2^{E_{i}}\right)^{2} \times \operatorname{BitCnt}(i)}}{\sum_{i^{\prime}=1}^{l}\left(\sqrt{\left(2^{E_{i^{\prime}}}\right)^{2} \times \operatorname{BitCnt}\left(i^{\prime}\right)}\right)}
\end{aligned}
$$

In Eq. (3), $E_{i}$ stands for the aforementioned exponent of the $i$-th row. Each column vector in matrix $A$ is the same, so $\left|A^{\left(i^{\prime}\right)}\right|$ equals to $\left|A^{(i)}\right|$. For the given $W$ with $l$ column vectors, $\sum_{i^{\prime}=1}^{l}\left|W_{\left(i^{\prime}\right)}\right|$ is a constant, so we let $C=\sum_{i^{\prime}=1}^{l}\left(\sqrt{\left.\left(2^{E_{i^{\prime}}}\right)^{2} \times \operatorname{BitCnt}\left(i^{\prime}\right)\right)}\right.$, and the final $p_{i}$ is deduced by the following Eq. (4):

$$
p_{i}=\frac{\sqrt{\left(2^{E_{i}}\right)^{2} \times \operatorname{BitCnt}(i)}}{C}
$$

Discussion: the probability $p_{i}$ reveals the magnitude of "significance". This is reasonable because $E_{i}$ reflects the bit significance of row $i$, and BitCnt $(i)$ reflects the number of essential bit $1 \mathrm{~s}$ in row $i$. Larger $E_{i}$ or BitCnt $(i)$ definitely leads to more contribution on the final MAC. BitX takes advantage of Eq. (4) to pinpoint the essential bit rows and in the meantime, prunes away the trivial bit rows directly in the accelerator.

\subsection{Pruning Procedure}

In Algorithm 1, BitX firstly interprets the exponent $E$ and mantissa $M$ of $\mathrm{n}$ fp32 weights as input (line $1 \sim 3$ ), aligns each exponent according to the $e_{\max }$ (line 4 ), and then calculates and sorts the row probabilities in descending order (line $5 \sim 12$ ). For the other input parameter $N$, it denotes the remaining bit rows in $W$ after pruning. In other words, BitX selects the top $n$ bit rows with relatively larger $p_{i} s$. The indices of the $n$ rows are reflected in $I^{\prime}$ (line 13). The pruning is finalized by the vector mask with the selected $n$ bit rows marked as ' 1 ' (line 7 and 16). Right after pruning, BitX extracts the essential bits and stores them into $W^{\prime}$ (line $17 \sim 23$ ).

The design parameter $N$ in the algorithm controls the granularity of pruning. Smaller $N$ inevitably leads to larger sparsity because more bit rows are pruned, which also benefits the inference by skipping more zero bits. In Section 4, we will thoroughly study the impact of $N$ on BitX performance.

\subsection{BitX Accelerator Architecture}

Bit $X$ is a hardware runtime pruning approach, so specially designed hardware pruning modules are integrated in the accelerator design. The overall full-system accelerator architecture is shown in Figure 3. Two module "E-alignment" and "Bit-Extraction" are designed to perform Algorithm 1. We instantiate 16 computing units to form one BitX PE. Each CU takes $M$ weights/activation pairs as input. The input weights are pre-processed by the "Bit-Extraction" module with the trivial bits pruned to 0 . CU executes the acceleration according to the significance of each bit in the pruned weight.

On our FPGA platform, the memory access is through DMA with two state machines to coordinate the data fetch and store, associated with the weight and activation buffer. Detailed FPGA and ASIC configuration are elaborated in Section 4.

For the fixed-point DNN, the E-alignment module is bypassed and safely powered down. Original weights are directly connected to the input of the Bit-Extraction module, because the fixed-point 
Table 4: Design space exploration of two key design parameters on ImageNet.

\begin{tabular}{|c|c|c|c|c|c|c|c|c|c|}
\hline \multirow{2}{*}{ ResNet50 } & \multicolumn{4}{|c|}{ Original Accuracy: 74.50} & \multirow{2}{*}{ DenseNet121 } & \multicolumn{4}{|c|}{ Original Accuracy: 71.96} \\
\hline & $\mathrm{N}=10$ & $\mathrm{~N}=8$ & $\mathrm{~N}=6$ & $\mathrm{~N}=4$ & & $\mathrm{~N}=10$ & $\mathrm{~N}=8$ & $\mathrm{~N}=6$ & $\mathrm{~N}=4$ \\
\hline$M=8$ & 74.50 & 74.51 & 74.10 & 67.00 & $M=8$ & 71.95 & 71.00 & 71.00 & 65.00 \\
\hline$M=16$ & 74.54 & 74.40 & 73.60 & 61.00 & $M=16$ & 71.97 & 72.00 & 71.00 & 62.00 \\
\hline$M=32$ & 74.00 & 74.50 & 73.50 & 58.20 & $M=32$ & 72.03 & 72.00 & 71.00 & 58.00 \\
\hline$M=64$ & 74.39 & 74.00 & 73.00 & 53.30 & $M=64$ & 71.00 & 71.00 & 70.00 & 55.00 \\
\hline$M=128$ & 74.41 & 74.32 & 72.70 & 46.30 & $M=128$ & 71.00 & 71.00 & 70.00 & 49.20 \\
\hline$M=256$ & 74.51 & 74.40 & 72.80 & 46.80 & $M=256$ & 71.84 & 71.00 & 69.00 & 49.00 \\
\hline$M=512$ & 74.30 & 74.26 & 71.90 & 39.40 & $\mathrm{M}=512$ & 71.83 & 71.60 & 69.00 & 34.00 \\
\hline \multirow{2}{*}{ ResNext101_32x8d } & \multicolumn{4}{|c|}{ Original Accuracy: 78.24} & SameezeNet1 1 & \multicolumn{4}{|c|}{ Original Accuracy: $\mathbf{5 4 . 8 4}$} \\
\hline & $\mathrm{N}=10$ & $\mathrm{~N}=8$ & $\mathrm{~N}=6$ & $\mathrm{~N}=4$ & Squeezenet1_1 & $\mathrm{N}=10$ & $\mathrm{~N}=8$ & $\mathrm{~N}=6$ & $\mathrm{~N}=4$ \\
\hline$M=8$ & 78.20 & 78.30 & 78.10 & 73.00 & $M=8$ & 54.86 & 54.70 & 54.40 & 47.30 \\
\hline$M=16$ & 78.20 & 78.00 & 77.50 & 66.00 & $M=16$ & 54.80 & 54.74 & 53.00 & 41.60 \\
\hline$M=32$ & 78.20 & 78.00 & 78.20 & 65.00 & $M=32$ & 54.00 & 54.50 & 53.64 & 41.70 \\
\hline$M=64$ & 78.20 & 78.20 & 77.30 & 62.00 & $M=64$ & 54.70 & 54.77 & 53.50 & 37.10 \\
\hline$M=128$ & 78.20 & 78.10 & 77.30 & 57.00 & $M=128$ & 54.40 & 54.48 & 52.80 & 34.66 \\
\hline$M=256$ & 78.20 & 78.20 & 77.20 & 49.00 & $M=256$ & 54.62 & 54.40 & 52.11 & 32.10 \\
\hline$M=512$ & 78.20 & 78.20 & 77.20 & 49.00 & $M=512$ & 54.81 & 54.72 & 52.60 & 32.00 \\
\hline
\end{tabular}

arithmetic does not involve exponent matching. Power gating is favorable to the abundant energy savings in the fixed-point BitX accelerator, as shown in the evaluations.

3.4.1 E-alignment. E-alignment module is designed to align the exponent of each weight uniformly to the maximum. It is mainly comprised of the data shifter and zero-padding. Firstly, the weight is split into the corresponding exponent and mantissa (for the floatingpoint data). Then, the maximum exponent $E_{\max }$ is obtained and stored. The exponents of all weights are aligned to this maximum value following Algorithm 1. The data shifter performs this operation through right shifting the $i$-th mantissa by $E_{\max }-E_{i}$. The shifted vacancies are zero-padded in the front part of the mantissa, marked as orange in Figure 3. For different weights, $E_{i}$ is possibly not identical, so we will obtain arbitrary bit widths after zero padding. To deal with this scenario, this module also pads a series of zero bits to the maximum bit width, marked as green in the figure. Although zero padding is frequent in this module, our RTL implementation could easily hard code this operation without violating the timing constraint. The only overhead introduced is the complicated wire organization that might potentially increase the circuit area.

3.4.2 Essential Bit Extraction. The padded mantissa output by the E-alignment module is then delivered to the Bit-Extraction module for the actual pruning. The $1^{\text {st }}$ functionality in this module is the BITCNT, which is designed to implement the BitCnt function in Eq. (4). In our FPGA implementation, the $\left(2^{E_{i}}\right)^{2} \times \operatorname{BitCnt}(i)$ operation inside SQRT could be equalized as shifting BitCnt $(i)$ by $2^{E_{i}}$. SQRT is not necessary because it will not influence the significance. Therefore, only combinatorial circuits could fulfill this purpose. The second functionality of the Bit-Extraction module is sorting the shifted BitCnt $(i)$ and selecting the top $\mathrm{n}$ largest rows, while the disqualified rows are completely pruned. The final pruned weight are thus obtained.
3.4.3 Compute Unit (CU). The pruned weights will surely exhibit more sparsity, and due to the pruning of the trivial bit ones, the left essential bits are substantially scarce. Therefore, a zero-skipping mechanism is designed in the "extractor" of the "Bit-Extraction" module to pinpoint the essential bits and further feed them to the compute unit (CU) module.

The microarchitecture of CU is shown in Figure 4. Each "selector" in the extractor targets one pruned binary weight ( $M$ weights in total), and $k$ denotes the bit in the pruned weight. The extractor records the significance of each essential bit $k$, represented as $s . s$ is used to shift the corresponding activation $A$ in each shifter.

The activations could be either floating-point or fixed-point data. The fixed-point $A$ could be directly shifted. However, for the floating-point $A$, the shifting operation is actually the exponent accumulation in $A$, which is the fixed-point arithmetic as well. Therefore, the shifters will not introduce severe overhead. The adder tree performs the final partial-sum accumulation. It distinguishes the precisions, so the overall power consumption of $\mathrm{CU}$ is also distinct under different precisions. Section 4.6 decomposes the power consumption of the BitX accelerator in ASIC design to give a comprehensive study.

\section{EVALUATION}

Benchmark and framework. The deep learning models and the parameters pre-trained for Cifar-10 [18] and ImageNet [15] dataset are directly obtained from PyTorch [1]. The benchmark models involve "big" models with the parameter size ranging from $76.35 \mathrm{M}$ (DenseNet201) to 356.71M (ResNext101_32x8d),as well as "little" models with the parameter size of 4.71M (SqueezeNet1_1). YoloV3 [28] trained on CoCo [14] dataset is employed to evaluate the performance on the object detection task. For the design parameter $\mathrm{N}$ and $\mathrm{M}$, we choose several discrete values for the design space exploration, to explore the sensitivity of BitX on the accuracy and speed. 


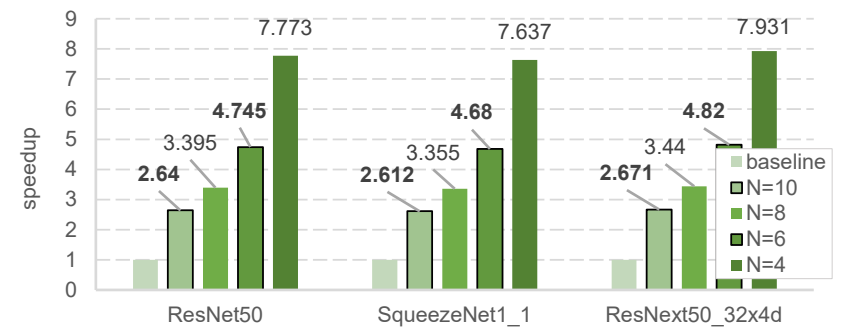

Figure 7: Inference speedup comparison under different $\mathbf{N}$ settings.

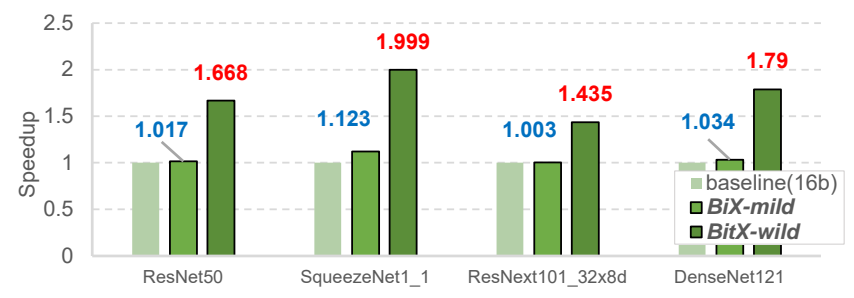

Figure 8: Inference speedup comparison of two BitX representatives on 16-bit fixed-point DNNs.

Also based on these parameters, we define two representatives of BitX - BitX-mild and BitX-wild.

FPGA \& ASIC implementation. At the RTL level, we employ Vivado HLS (v2018.2) to conduct post-synthesis simulation on Xilinx Virtex-7 FPGA. The actual inference time is recorded at each run. We instantiate $16 \mathrm{CUs}$ in PE, clocked at $200 \mathrm{MHz}$. Runtime memory access data of our FPGA platform are recorded and then fed to the DRAMsys tool [17] to estimate the energy consumption of the memory accesses. For ASIC, Synopsis Design Compiler (v2016) is used to measure power and area. The frequency is set to 1 $\mathrm{GHz}$. The whole design is synthesized with TSMC 28nm technology library.

\subsection{Accuracy \& Sparsity}

4.1.1 Cifar-10. Table 2 shows the accuracy/sparsity results for Cifar-10 dataset, grouped by the parameter $N$. Smaller $N$ means more bit rows are pruned, so the bit-level sparsity also turns larger. For example for $N=4$, the sparsity increases to $1.80 \mathrm{x}$ compared with the original model. More sparsity is undoubtedly beneficial to the inference speedup (as proved in Section 4.2). On the other hand, larger $N$ means less bit rows are pruned so the sparsity only shows $1.41 \mathrm{x}$ and $1.53 \mathrm{x}$ for $N=10$ and $N=8$.

Bit $X$ shows promising pruning accuracy. The average accuracy loss is about $0.1 \%$ at $N=10,8$ and 6 for various evaluated DNNs.

4.1.2 ImageNet. The results for ImageNet dataset shown in Table 3 exhibit a similar trend as Table 2: less than $0.5 \%$ average accuracy loss at $N=10,8,6$, and 1.40x, 1.52x, 1.66x sparsity increment apiece.

Discussion: firstly, it proves that the proposed BitX pruning methodology will not affect the accuracy of DNNs. The average accuracy loss is less than $0.5 \%$ at $N=10,8$ and 6, for both Cifar-10 and ImageNet datasets. Secondly, this experiment clearly demonstrates the tradeoff between accuracy and sparsity. A borderline
Table 5: ImageNet performance of two BitX representatives under 16-bit fixed-point DNNs.

\begin{tabular}{llll}
\hline Model & Baseline(16b) & Bit $\boldsymbol{X}$-mild & Bit $\boldsymbol{X}$-wild \\
\hline ResNet50 & 74.50 & 74.50 & 74.10 \\
& & $\mathbf{( 0 . 0 0 )}$ & $(-0.40)$ \\
SqueezeNet1_1 & 54.86 & 54.80 & 54.40 \\
& & $(-0.06)$ & $(-0.46)$ \\
DenseNet121 & 71.00 & 71.90 & $\mathbf{7 1 . 8 0}$ \\
& & $\mathbf{( + 0 . 9 0 )}$ & $\mathbf{( + 0 . 8 0 )}$ \\
ResNext101_32x8d & 78.00 & $\mathbf{7 8 . 2 0}$ & $\mathbf{7 8 . 1 0}$ \\
& & $(+\mathbf{0 . 2 0 )}$ & $\mathbf{( + 0 . 1 0 )}$ \\
\hline
\end{tabular}

configuration also exists with the maintained accuracy and satisfied sparsity. As shown in Table 2 and Table 3, there is a significant accuracy drop at $N=4$ and $N=6$. Therefore, we can safely choose $N$ values in the range $10 \sim 6$ in BitX. This experiment also verifies that there are tremendously redundant bits in the parameters that can be safely pruned without hurting the accuracy.

\subsection{Speedup}

We evaluate the inference speed at different sparsity levels indicated by the $N$ configuration. The speedup data are recorded according to the actual inference cycles on our Xilinx V7 FPGA platform and normalized to the original non-pruned DNN. As shown in Figure 7, BitX exhibits $\sim 2.6 \mathrm{x}$ speedup at $N=10$, and $\sim 4.8 \mathrm{x}$ speedup at $N=6$. The promising speedup stems from the enriched bit sparsity enforced by the Bit $X$ pruning. More abundant sparsity enables more zero-bit skipping in the BitX accelerator, and thus leads to much faster inference speed.

Discussion: BitX accelerator directly integrates the pruning module in hardware, and executes hardware runtime pruning during inference. This is totally different from software-based pruning that targets value sparsity to acquire reduced parameter size and FLOPs. BitX leverages the abundant useless bits to directly accelerate the original DNN after deployment, and does not involve any software work. The high speedup and lossless accuracy can provide attractive convenience for the end users to deploy their models into products much faster.

\subsection{Design Space Exploration}

Section 4.1 and Section 4.2 have evaluated the sensitivity of $N$ and its impact to the BitX performance. We further explore the impact of another key parameter $M$ in this experiment. We use $4 \mathrm{DNN}$ trained with the ImageNet dataset as shown in Table $4 . M$ indicates the number of input weights that the accelerator could simultaneously prune (Figure 3), and generally speaking, $M$ barely influences the overall accuracy scaling from $8 \sim 512$ for all the $4 \mathrm{DNNs}$. For example, in ResNet50 the accuracy at $M=8$ is lower than the accuracy at $M=16$ but is higher than the accuracy at $M=32$ or 64 . For SqueezeNet1_1, the accuracy at $M=8(54.86 \%)$ is even higher than the original model accuracy $(54.84 \%)$. The average accuracy loss at other $M$ configurations is less than $0.3 \%$. We conclude that the number of simultaneous input weights has negligible impact to the performance of BitX. 
Table 6: Performance of BitX collaborating with softwarebased pruning. We use the genetic and channel-pruned YoloV3 model.

\begin{tabular}{|c|c|c|}
\hline Method & $\operatorname{mAP}(\%)$ & Speedup $(\mathbf{x})$ \\
\hline YoloV3 (baseline) & 50.36 & 1 \\
\hline YoloV3 + BitX-mild & $\begin{array}{l}(50.42) \\
(+0.06)\end{array}$ & 2.75 \\
\hline YoloV3 + BitX-wild & $\begin{array}{l}50.05 \\
(-0.31)\end{array}$ & 4.98 \\
\hline YoloV3 + Slimming [22] (baseline) & $\begin{array}{l}50.23 \\
(-0.13)\end{array}$ & 2.35 \\
\hline YoloV3 + Slimming [22] + BitX-mild & $\begin{array}{l}50.30 \\
(+0.07)\end{array}$ & 7.22 \\
\hline YoloV3 + Slimming [22] + BitX-wild & $\begin{array}{l}48.72 \\
(-1.64)\end{array}$ & 14.76 \\
\hline
\end{tabular}
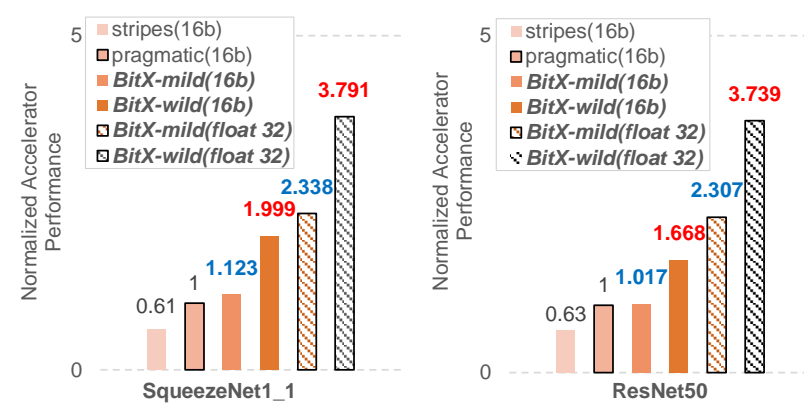

Figure 9: Speedup comparison with other SOTA accelerators.
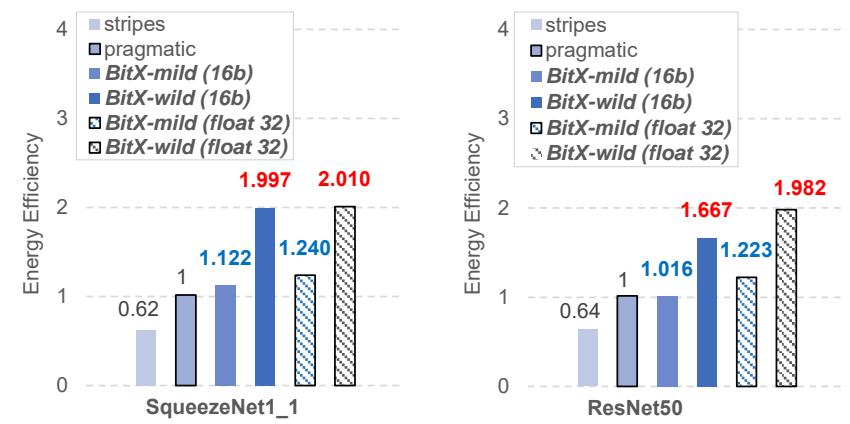

Figure 10: Energy efficiency comparison. Higher is better.

Discussion: the major factor that steers the accuracy and speedup is the $N$ configuration. Table 4 shows that at different scales of $M$, the accuracy consistently degrades from $N=10$ to $N=4$, which is in line with the observation in Table 3 . It is $N$ that decides the granularity of pruning, while $M$ only controls the input throughput.

Two BitX instances: as discussed above, $M$ barely influences the accuracy, so we choose $M=8$ for the efficient accelerator implementation. Upon $M=8$, we select two $N$ settings: $N=10$ and $N=6$ to form two BitX instances, termed as BitX-mild ( $N=10$, $M=8)$ and BitX-wild $(N=6, M=8)$. BitX-mild has the topmost accuracy but limited speedup, while BitX-wild has little-degraded

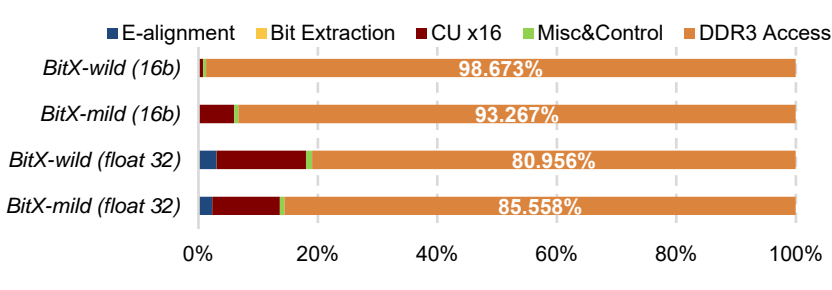

(a) Full system energy breakdown

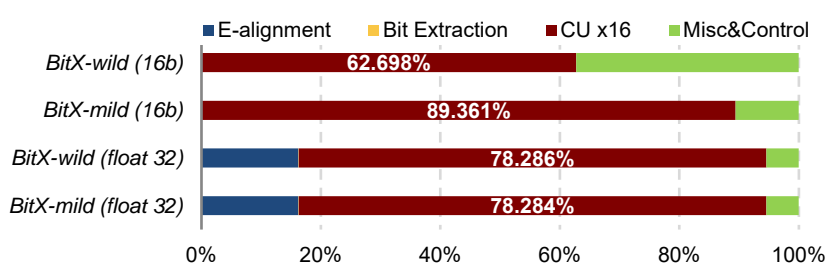

(b) BitX PE-only energy breakdown

Figure 11: full-system and PE-only energy breakdown for SqueezeNet.

Table 7: PE area and power breakdown @TSMC 28nm.

\begin{tabular}{llll}
\hline Precision & $\begin{array}{l}\text { BitX } \\
\text { (floating-point 32) }\end{array}$ & $\begin{array}{l}\text { BitX } \\
\text { (16b fixed point) }\end{array}$ \\
\hline \multirow{2}{*}{ Item } & $\begin{array}{l}\text { Area } \\
\left(\mathbf{m m}^{2}\right)\end{array}$ & $\begin{array}{l}\text { Power } \\
(\mathbf{m W})\end{array}$ & $\begin{array}{l}\text { Power } \\
\text { (mW) }\end{array}$ \\
\hline E-alignment & 0.017 & 11.15 & \\
& $(43.60 \%)$ & $(16.20 \%)$ & \\
Bit Extraction & 0.008 & 0.04 & 0.026 \\
& $(20.10 \%)$ & $(0.05 \%)$ & $(0.07 \%)$ \\
16 CUs & 0.003 & 53.71 & 35.81 \\
& $(7.70 \%)$ & $(78.30 \%)$ & $(98.40 \%)$ \\
Misc\&Control & 0.011 & 3.72 & 0.576 \\
& $(28.20 \%)$ & $(5.40 \%)$ & $(1.60 \%)$ \\
Total & $\mathbf{0 . 0 3 9}$ & $\mathbf{6 8 . 6 2}$ & $\mathbf{3 6 . 4 1}$ \\
\hline
\end{tabular}

accuracy but relatively abundant speedup. Two instances are used to verify the "accuracy-speedup" tradeoff, and in the rest of this section, we will use the two instances as the representatives of BitX to compare with other SOTA accelerator baselines.

\subsection{Performance of the Fixed-point DNN}

Accuracy. BitX is also feasible to $16 \mathrm{~b}$ fixed-point DNNs, as part of its versatility. Fixed-point weight also exhibits substantial useless bits for pruning, but the difference with floating-point weight is that it does not need exponent matching. Therefore, the "E-alignment" module in BitX accelerator is not needed and could be power gated (Figure 3). The weights directly pass through to the "Bit-Extraction" module for sorting the probabilities of each bit row. As Table 5 shows, the accuracy is exactly equal to the non-pruned ResNet50 for BitX-mild. More promisingly, BitX-mild and BitX-wild both 
exhibit even higher accuracy than the non-pruned DenseNet121 and ResNext101.

The accuracy improvement attains up to nearly $1 \%$. We conclude that BitX could precisely pinpoint the useless bits in both floating-point and fixed-point DNNs.

Speedup. As shown in Figure 8, BitX-wild exhibits up to $2 \mathrm{x}$ speedup over the original model. Reporting some of the results, for ResNet50, the speedup on BitX-wild is $1.67 \mathrm{x}$; for DenseNet121, the datum is $1.79 \mathrm{x}$. As for the BitX-mild, the largest speedup emerges at SqueezeNet1_1 - 1.12x. Other DNNs demonstrate tiny acceleration, primarily because each weight only has 16-bit width, setting $N=10$ means only 6-bit width is pruned. The trivial bit 1 s pruned are very limited. By sharp contrast, BitX-wild will prune 10 bits for each weight. Hence, the speedup is abundant.

\subsection{Working with Software-based Pruning}

As a hardware runtime pruning approach, BitX is orthogonal to any software-based pruning schemes. In this experiment, we use the famous object detection model - YoloV3 [28] as the benchmark DNN, including the genetic YoloV3 model and software-pruned model based on the structured channel pruning [22]. As presented in Table 6, BitX-mild still displays better performance than the genetic YoloV3 with $2.75 \mathrm{x}$ speedup. BitX-wild exhibits tiny-degraded accuracy but higher speedup - 4.98x. For the YoloV3+Slimming baseline, Bit-mild has $0.07 \%$ accuracy improvement and BitX-wild has less than $1.6 \%$ accuracy degradation. The speedup is very much considerable over the genetic YoloV3: YoloV3+Slimming+BitX-wild attains 14.76x; YoloV3+Slimming+BitX-mild attains 7.22x. This experiment clearly proves that our method is completely compatible to the previous software pruning schemes. The users could effortlessly obtain more superior speedup and accuracy by collaborating BitX with software pruning.

\subsection{Comparison with SOTA Accelerators}

In this subsection, we compare the two BitX representatives with the state-of-the-art fixed-point accelerators. Stripes [16] and Pragmatic [2] are two bit-serial accelerators. Stripes implements the MAC computation using bit-level arithmetic, but does not consider the sparsity. Pragmatic, on top of stripes, exploits the bit sparsity by dynamically skipping the zero bits. However, it is not designed for bit pruning. BitX targets two types of useless bits. The trivial bit $1 \mathrm{~s}$ are also pruned which means the amount of 0 bits that can be skipped becomes more, hence yielding better speedup performance.

Speedup. As proved by Figure 9, the speedup over Pragmatic (normalized baseline) and stripes is 2.00x and 3.79x for BitX-wild; and $1.12 \mathrm{x}, 2.34 \mathrm{x}$ for BitX-mild. A more interesting observation is that the floating-point results are even better than the fixed-point BitX, still because of the limited 16-bit width. Floating-point weight has 24-bit mantissa and spawns even more useless bits after exponent matching. The functional flexibility provided by Bit $X$ also releases more AI tasks that could run on BitX. The users could freely customize their DNNs in the practical use.

Energy efficiency. Similar to the speedup result, the energy efficiency of BitX outperforms other accelerator baselines, but this time the 16b BitX-wild (2.00x, SqueezeNet, 1.68x ResNet50) demonstrates better result than the float-32 BitX-mild $(1.24 \mathrm{x}, 1.22 \mathrm{x})$. That is because the floating-point BitX has higher power consumption due to the "E-alignment" module which is power gated in the $16 \mathrm{~b}$ mode. However, combined with the inference speed, BitX-wild (float 32) still wins for 2.01x and 1.98x better efficiency.

Energy breakdown. Our Xilinx V7 FPGA platform involves DDR3 memory. We use DRAMsys to estimate the runtime memory access energy. Figure 11 shows the energy breakdown from two aspects. Figure 11(a) shows the full-system energy breakdown and clearly the memory accesses dominate the energy consumption. Especially for the two 16-bit BitX representatives, the memory access energy could attain $98 \%$ and PE energy only occupies less than $2 \%$. In Figure 11(b), we further decompose the PE-only energy for each BitX instance. CU energy dominates this time $(63 \%, 89 \%$, $78 \%, 78 \%$ ), because we have 16 CUs with a large number of buffers to store the bit-pruned weights. For other modules, E-alignment and the control circuits consume around 16\% and 5\% 37\% energy, respectively.

Area and Power breakdown. Under TSMC $28 \mathrm{~nm}$ technology node, BitX in floating-point 32 mode exhibits $0.039 \mathrm{~mm}^{2}$ area. Table 7 illustrates the largest area is occupied by the E-alignment module (43.6\%), because it involves frequent shifting operation and some of the wires are inevitably prolonged to avoid intersection. However, it is not the largest power consumer (only 16.2\%) because no computation circuits are involved in this module. Comparatively, the 16 CUs occupy the smallest area (7.7\%) but consume most of the power $(78.3 \%)$ due to the internal arithmetic logic. BitX in 16-bit fixed-point mode powers down the E-alignment module and the $\mathrm{CU}$ arithmetic is based on the fixed-point activations, so its overall power consumption reduces to $36.41 \mathrm{~mW}$, compared with 68.62 $\mathrm{mW}$ in the floating-point 32 mode.

\section{CONCLUSION}

In this paper, we propose a novel hardware runtime pruning method - BitX, to empower versatile DNN inference. By targeting the abundant bit-level sparsity and trivial bit $1 \mathrm{~s}$, it implements pruning on-the-fly in hardware without any software work. It precisely locates the essential bits by the proposed BitX pruning algorithm, and prunes away the trivial bits at different precisions including both floating point and fixed point. The empirical studies have proved the efficacy of BitX, by providing abundant sparsity, faster inference speed and lossless (or even higher) accuracy on various image classification and object detection models. We also hope BitX pruning methodology and the associate accelerator design would stimulate more insightful perspectives on hardware runtime pruning, to provide both promising DNN acceleration and excellent user experience at the same time.

\section{ACKNOWLEDGMENTS}

This work is supported in part by the National Natural Science Foundation of China under grant No. 62002339 and grant No. U2030204, and the Strategic Priority Research Program of Chinese Academy of Sciences under grant No. XDB44030200, and the Key Research Program of State Key Laboratory of Computer Architecture under grant No. CARCH5301. 


\section{REFERENCES}

[1] pytorch 1.6. https://pytorch.org/

[2] Jorge Albericio, Patrick Judd, Alberto Delmas, Sayeh Sharify, and Andreas Moshovos. 2016. Bit-pragmatic Deep Neural Network Computing. In Proceedings of the International Symposium on Microarchitecture (MICRO).

[3] Jorge Albericio, Patrick Judd, Tayler Hetherington, Tor Aamodt, Natalie Enright Jerger, and Andreas Moshovos. 2016. Cnvlutin: Ineffectual-Neuron-Free Deep Neural Network Computing. In Proceedings of the International Symposium on Computer Architecture (ISCA).

[4] Sajid Anwar, Kyuyeon Hwang, and Wonyong Sung. 2015. Structured Pruning of Deep Convolutional Neural Networks. ACM fournal on Emerging Technologies in Computing Systems (JETC).

[5] Tom B. Brown, Benjamin Mann, Nick Ryder, Melanie Subbiah, Jared Kaplan, Prafulla Dhariwal, Arvind Neelakantan, Pranav Shyam, Girish Sastry, Amanda Askell, Sandhini Agarwal, Ariel Herbert-Voss, Gretchen Krueger, Tom Henighan, Rewon Child, Aditya Ramesh, Daniel M. Ziegler, Jeffrey Wu, Clemens Winter, Christopher Hesse, Mark Chen, Eric Sigler, Mateusz Litwin, Scott Gray, Benjamin Chess, Jack Clark, Christopher Berner, Sam McCandlish, Alec Radford, Ilya Sutskever, and Dario Amodei. 2020. Language Models are Few-Shot Learners $\operatorname{arXiv:2005.14165.}$

[6] Jifeng Dai, Haozhi Qi, Yuwen Xiong, Yi Li, Guodong Zhang, Han Hu, and Yichen Wei. 2017. Deformable Convolutional Networks. In Proceedings of the Conference on Computer Vision and Pattern Recognition (CVPR).

[7] Jacob Devlin, Ming-Wei Chang, Kenton Lee, and Kristina Toutanova. 2018. BERT: Pre-training of Deep Bidirectional Transformers for Language Understanding. arXiv:1810.04805.

[8] Petros Drineas, Ravi Kannan, and Michael W Mahoney. 2006. Fast Monte Carlo Algorithms for Matrices III: Computing a Compressed Approximate Matrix Decomposition. SIAM fournal on Computing (SICOMP).

[9] Song Han, Junlong Kang, Huizi Mao, Yiming Hu, Xin Li, Yubin Li, Dongliang Xie Hong Luo, Song Yao, Yu Wang, Huazhong Yang, and William J. Dally. 2016. ESE: Efficient Speech Recognition Engine with Sparse LSTM on FPGA. In Proceedings of the ACM/SIGDA International Symposium on Field-Programmable Gate Arrays (FPGA).

[10] Kaiming He, Xiangyu Zhang, Shaoqing Ren, and Jian Sun. 2016. Deep residual learning for image recognition. In Proceedings of the Conference on Computer Vision and Pattern Recognition (CVPR).

[11] Hengyuan Hu, Rui Peng, Yu-Wing Tai, and Chi-Keung Tang. 2016. Network trimming: A data-driven neuron pruning approach towards efficient deep architectures. arXiv:1607.03250.

[12] Gao Huang, Zhuang Liu, Laurens van der Maaten, and Kilian Q. Weinberger. 2017 Densely Connected Convolutional Networks. In Proceedings of the Conference on Computer Vision and Pattern Recognition (CVPR).

[13] IEEE 754. https://standards.ieee.org/standard/754-2019.html

[14] CoCo Dataset. https://cocodataset.org/

[15] Jia Deng, Wei Dong, Richard Socher, Li-Jia Li, Kai Li, and Fei-Fei Li. 2009. ImageNet: A large-scale hierarchical image database. In Proceedings of the Conference on Computer Vision and Pattern Recognition (CVPR).

[16] Patrick Judd, Jorge Albericio, Tayler Hetherington, Tor M. Aamodt, and Andreas Moshovos. 2017. Stripes: Bit-Serial Deep Neural Network Computing. In Proceedings of the International Symposium on Microarchitecture (MICRO).

[17] Matthias Jung, Christian Weis, and Norbert Wehn. 2015. DRAMSys: A Flexible DRAM Subsystem Design Space Exploration Framework. IPSf Transactions on System LSI Design Methodology (T-SLDM).
[18] Alex Krizhevsky and Geoff Hinton. 2009. Learning multiple layers of features from tiny images. In Proceedings of the Conference on Computer Vision and Pattern Recognition (CVPR).

[19] Alberto Delmas Lascorz, Patrick Judd, Dylan Malone Stuart, Zissis Poulos, and Mostafa Mahmoud. 2019. Bit-Tactical: A Software/Hardware Approach to Exploiting Value and Bit Sparsity in Neural Networks. In Proceedings of the Architectural Support for Programming Languages and Operating Systems (ASPLOS).

[20] Jinmook Lee, Changhyeon Kim, Sanghoon Kang, Dongjoo Shin, and Hoi Jun Yoo. 2018. UNPU: A 50.6 TOPS/W unified deep neural network accelerator with 1bto-16b fully-variable weight bit-precision. In Proceedings of the IEEE International Solid-State Circuits Conference (ISSCC).

[21] Hao Li, Asim Kadav, Igor Durdanovic, Hanan Samet, and Hans Peter Graf. 2017. Pruning filters for efficient convnets. In Proceedings of the International Conference on Learning Representations (ICLR).

[22] Zhuang Liu, Jianguo Li, Zhiqiang Shen, Gao Huang, Shoumeng Yan, and Changshui Zhang. 2017. Learning Efficient Convolutional Networks through Network Slimming. In Proceedings of the IEEE International Conference on Computer Vision (ICCV).

[23] Hang Lu, Xin Wei, Ning Lin, and Guihai Yan. 2018. Tetris: Re-architecting Convolutional Neural Network Computation for Machine Learning Accelerators. In Proceedings of the International Conference On Computer Aided Design (ICCAD).

[24] Hang Lu, Mingzhe Zhang, Yinhe Han, Qi Wang, Huawei Li, and Xiaowei Li. 2020. Architecting Effectual Computation for Machine Learning Accelerators.
IEEE Transactions on Computer-Aided Design of Integrated Circuits and Systems (TCAD).

[25] Jian-Hao Luo and Jianxin Wu. 2017. An entropy-based pruning method for CNN compression. arXiv:1706.05791.

[26] Jian-Hao Luo, Jianxin Wu, and Weiyao Lin. 2017. ThiNet: A Filter Level Pruning Method for Deep Neural Network Compression. In Proceedings of the IEEE International Conference on Computer Vision (ICCV).

[27] Angshuman Parashar, Minsoo Rhu, Anurag Mukkara, Antonio Puglielli, Rangharajan Venkatesan, Brucek Khailany, Joel Emer, Stephen W. Keckler, and William J. Dally. 2017. SCNN: An Accelerator for Compressed-sparse Convolutional Neural Networks. In Proceedings of the International Symposium on Computer Architecture (ISCA)

[28] Joseph Redmon and Ali Farhadi. 2018. YOLOv3: An Incremental Improvement. In Proceedings of the Conference on Computer Vision and Pattern Recognition (CVPR).

[29] Karen Simonyan and Andrew Zisserman. 2015. Very deep convolutional networks for large-scale image recognition. arXiv:1409.1556.

[30] Song Han, Xingyu Liu, Huizi Mao, Jing Pu, and William J. Dally. 2016. EIE: Efficient Inference Engine on Compressed Deep Neural Network. In Proceedings of the International Symposium on Computer Architecture (ISCA).

[31] Du Tran, Lubomir Bourdev, Rob Fergus, Lorenzo Torresani, and Manohar Paluri. 2014. Learning Spatiotemporal Features with 3D Convolutional Networks. arXiv:1412.0767.

[32] Xiaolong Wang, Ross Girshick, Abhinav Gupta, and Kaiming He. 2014. Non-local Neural Networks. In Proceedings of the Conference on Computer Vision and Pattern Recognition (CVPR).

[33] Wei Wen, Chunpeng Wu, Yandan Wang, Yiran Chen, and Hai Li. 2016. Learning Structured Sparsity in Deep Neural Networks. In Proceedings of the Conference and Workshop on Neural Information Processing Systems (NeurIPS).

[34] Xuda Zhou, Zidong Du, Qi Guo, Shaoli Liu, and Yunji Chen. 2018. CambriconS: Addressing Irregularity in Sparse Neural Networks through A Cooperative Software/Hardware Approach. In Proceedings of the International Symposium on Microarchitecture (MICRO). 